\title{
Optical coherence tomography guided successful fibrinolytic treatment without the need for percutaneous coronary intervention in a patient with acute ST-segment elevation myocardial infarction
}

\author{
Onur Tasar ${ }^{1}$, Arzu Kalayci², Mehmet Emin Kalkan², Can Yucel Karabay², Gönenç Kocabay², Cevat Kirma² \\ ${ }^{1}$ Department of Cardiology, Elazig Research and Education Hospital, Elazig, Turkey \\ ${ }^{2}$ Department of Cardiology, Kosuyolu Heart Center, Elazig, Turkey
}

Adv Interv Cardiol 2018; 14, 2 (52): 199-201

DOI: https://doi.org/10.5114/aic.2018.76414

A 36-year-old woman was admitted to the coronary care unit with a diagnosis of myocardial infarction with ST-segment elevation (STEMI) after an hour of chest pain. She was a smoker and she had no history of any other atherosclerotic risk factors. She had a stable haemodynamic status without signs of heart failure. An electrocardiogram was obtained, revealing ST-segment elevation in anterior leads and reciprocal changes in inferior leads. Given the ST-segment elevation in the context of new-onset chest pain, she was given 7500 IU of unfractionated heparin, $300 \mathrm{mg}$ of aspirin, and $600 \mathrm{mg}$ of clopidogrel and taken immediately for coronary angiography (CAG). Coronary angiography revealed that there was a large filling defect in the proximal left anterior descending coronary artery (LAD) consistent with thrombus with TIMI grade II distal flow (Figures $1 \mathrm{~A}, \mathrm{~B}$ ). We did not perform either wiring or thrombus aspiration due to high risk of thrombus shift into the distal LAD and circumflex artery, which may cause the no-reflow phenomenon. According to our experience, we decided to manage this large thrombus burden only using pharmacological therapy. Intracoronary administration of alteplase (rt-PA) as a $15 \mathrm{mg}$ bolus and intravenous infusion of $50 \mathrm{mg}$ for $30 \mathrm{~min}$ were applied. Chest pain was relieved and ST segment resolution occurred after fibrinolytic therapy. Control CAG and intracoronary optical coherence tomography (OCT) were performed $8 \mathrm{~h}$ later. Coronary angiography revealed partially dissolved thrombus with TIMI grade III distal flow (Figure $1 \mathrm{C}$ ). Optical coherence tomography depicted better partially dissolved red residual thrombus (Figure $1 \mathrm{D}$ ).
Additional $50 \mathrm{mg}$ rt-PA was infused intravenously for $12 \mathrm{~h}$ to dissolve the residual thrombus. Seventy-two hours later, we performed the second control CAG and OCT. Repeated CAG revealed no visible residual thrombus with TIMI grade III distal flow (Figure $1 \mathrm{E}$ ), whereas OCT showed definite plaque erosion and a small organized thrombus attached to the arterial wall. No plaque rupture was visualized (Figure $1 \mathrm{~F}$ ). After administration of rt-PA twice, which is the first time in the literature, we did not deploy a stent. The patient was discharged with optimal medical therapy including aspirin, clopidogrel, metoprolol and statin. Follow-up was uneventful at one year.

Patients presenting with STEMI have many different etiologies of coronary occlusion. In clinical and autopsy series, $15-30 \%$ of patients with acute coronary syndrome (ACS) have coronary thrombosis due to plaque erosion. Optical coherence tomography can provide images which enable us to assess different pathologic features such as cap thickness, plaque erosion and rupture $[1,2]$. Prior studies suggest different natural histories and outcomes for patients with plaque erosion, since little underlying stenosis or flow limitation exists. The patients are frequently women and active smokers like our patient [3].

Limited serial OCT studies indicate that medical treatment may change the lesion characteristics where the lesion may be deferred for spontaneous healing instead of intervention $[4,5]$. Although we need large studies in this special patient group, our case presents an alternative approach for the treatment of ACS. 

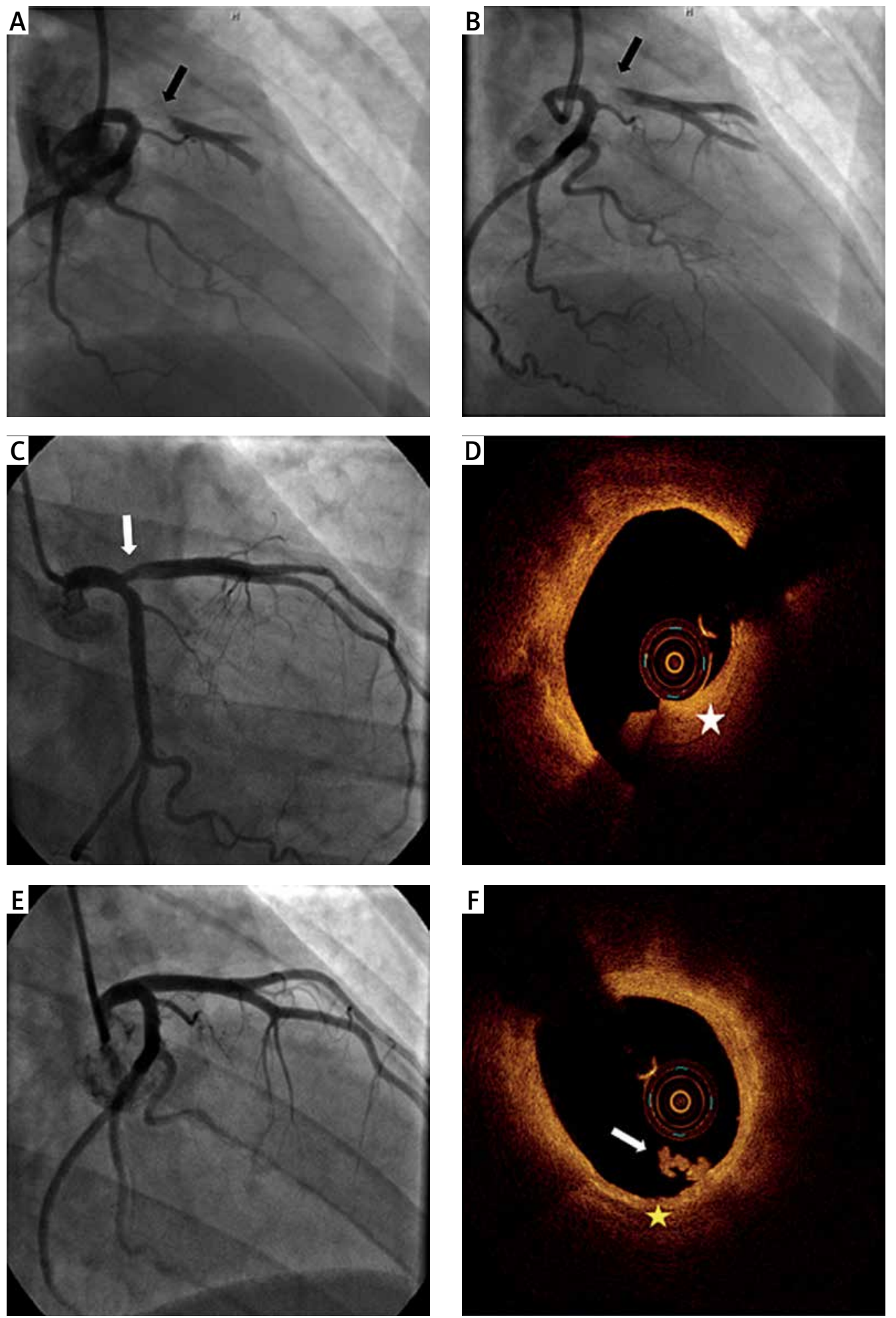

Figure 1. Serial coronary angiography and optical coherence tomography. A, B - Initial angiography revealed a large thrombus (black arrow) in the proximal left anterior descending artery (LAD). C - Eight hours after the first alteplase intracoronary bolus and infusion, coronary angiography revealed a small thrombus-like image (yellow arrow) in the proximal LAD. D - Eight hours after the first alteplase intracoronary bolus and infusion, optical coherence tomography (OCT) revealed a red thrombus (white star) in the proximal LAD lumen. $\mathbf{E}-\mathrm{At}$ $72 \mathrm{~h}$ after the second alteplase infusion, repeated coronary angiography revealed no residual thrombus in the LAD. F - At $72 \mathrm{~h}$ after the second alteplase infusion, OCT revealed plaque erosion (yellow star) and a small attached organized thrombus (white arrow) in the proximal LAD 


\section{Conflict of interest}

The authors declare no conflict of interest.

\section{References}

1. Giannopoulos AA, Antoniadis AP, Croce K, et al. Erosion of thincap fibroatheroma in an area of low endothelial shear stress: anatomy and local hemodynamic environment dictate outcomes. JACC Cardiovasc Interv 2016; 9: e77-8.

2. Pepine CJ, Ferdinand KC, Shaw LJ, et al. Emergence of nonobstructive coronary artery disease: a woman's problem and need for change in definition on angiography. J Am Coll Cardiol 2015; 66: 1918-33.

3. Higuma T, Soeda T, Abe N, et al. A combined optical coherence tomography and intravascular ultrasound study on plaque rupture, plaque erosion, and calcified nodule in patients with ST-segment elevation myocardial infarction: incidence, morphologic characteristics, and outcomes after percutaneous coronary intervention. JACC Cardiovasc Interv 2015; 8: 1166-76.

4. Hu S, Zhu Y, Zhang Y, et al. Management and outcome of patients with acute coronary syndrome caused by plaque rupture versus plaque erosion: an intravascular optical coherence tomography study. J Am Heart Assoc 2017; 6: pii: e004730.

5. Kwon JE, Lee WS, Mintz GS, et al. Multimodality intravascular imaging assessment of plaque erosion versus plaque rupture in patients with acute coronary syndrome. Korean Circ J 2016; 46: 499-506. 